\title{
An Improved Variable Structure Adaptive Filter Design and Analysis for Acoustic Echo Cancellation
}

\author{
Asutosh KAR ${ }^{1}$, Mahesh CHANDRA ${ }^{2}$ \\ ${ }^{1}$ Dept. of ECE, IIIT, Bhubaneswar, Odisha, India-751003 \\ ${ }^{2}$ Dept. of ECE, BIT, Mesra, Ranchi, Jharkhand, India- 835215 \\ asutosh@iiit-bh.ac.in, shrotriya@bitmesra.ac.in
}

\begin{abstract}
In this research an advanced variable structure adaptive Multiple Sub-Filters (MSF) based algorithm for single channel Acoustic Echo Cancellation (AEC) is proposed and analyzed. This work suggests a new and improved direction to find the optimum tap-length of adaptive filter employed for AEC. The structure adaptation, supported by a tap-length based weight update approach helps the designed echo canceller to maintain a trade-off between the Mean Square Error (MSE) and time taken to attain the steady state MSE. The work done in this paper focuses on replacing the fixed length sub-filters in existing MSF based AEC algorithms which brings refinements in terms of convergence, steady state error and tracking over the single long filter, different error and common error algorithms. A dynamic structure selective coefficient update approach to reduce the structural and computational cost of adaptive design is discussed in context with the proposed algorithm. Simulated results reveal a comparative performance analysis over proposed variable structure multiple sub-filters designs and existing fixed tap-length sub-filters based acoustic echo cancellers.
\end{abstract}

\section{Keywords}

Adaptive filter, acoustic echo cancellation, variable structure design, multiple sub-filters, least mean square, mean square error, convergence

\section{Introduction}

Adaptive system identification designs fix the filter length at some compromise value which makes it ineffective in dynamic echo cancellation environments [1], [2]. The desired optimum tap-length best balances the complexity and steady state performance of the adaptive filter in time-varying echoed environment [3-6]. In practice the length or order of the plant which is to be identified by the adaptive filter is not known previously and has to be estimated from the knowledge of input and output signals. In Acoustic Echo Cancellation (AEC) the room impulse response varies from a small room impulse response to a large room impulse response. All conversations whether face to face or over a communication network, generates echo. However, if the time delay corresponding to the acoustic loop is small then significant echo is not observed [2]. The problem is well defined in case of hands-free communication. The presence of large acoustic coupling between the loudspeaker and microphone would produce an echo that causes a reduction in the quality of the communication. Hence, normally linear adaptive filters carrying thousands of weights are employed to form an echo cancellation framework. This large order filters result in high complexity and adaptation noise [3-6]. The Least Mean Square (LMS) algorithm in general depicts slow convergence in AEC. This happens due to the long length and large response of the acoustic echo channel [7].

In this research work a set of Variable Structure Multiple Sub-Filters (VS-MSF) algorithms are discussed where a Single Long Filter (SLF) is subdivided into number of sub-filters and order of each sub-filter is optimized as per the echoed environment [8-10]. The steady state Mean Square Error (MSE) accomplishment of adaptive Multiple Sub-Filters (MSF) Different Error Algorithm (DEA) is poor compared to MSF Common Error Algorithm (CEA) and adaptive SLF for AEC [9]. On the other hand the performance degradation in MSE for DEA is compensated by a fast convergence due to individualistic contemplation of each error signal. Similarly the CEA results in better steady state error than SLF and DEA but the speed to achieve optimized MSE decreases [10-12].

A combination of both the MSF DEA and MSF CEA is studied to come with an improved methodology for echo cancellation. It holds the benefits of both the existing MSF algorithms and as it is a combination of DEA as well as CEA has been mentioned as Combined Error Algorithm (COEA) [9]. In this paper this MSF COEA based acoustic echo canceller is implemented with a structure adaptation criterion to attain improved results with minimized structural and computational intricacy. The improvements of the proposed Variable Structure Multiple Sub-Filters based Combined Error Algorithm (VS-MSF-COEA) and its implementation is compared with the Variable Structure Multiple Sub-Filters based Different Error Algorithm (VSMSF-DEA) and Variable Structure Multiple Sub-Filters based Common Error Algorithm (VS-MSF-CEA). This proposed design not only leads to significant improvement 
in convergence rate but also brings refinement in steady state error. The rendition of these VS-MSF based echo cancellers is compared with regular echo cancellers in which a Variable Structure Single Long Filter (VS-SLF) is used to model the response of the acoustic channel [11].

A way to diminish the computational entanglement of adaptive algorithms is shown in which only a particular sub set of all filter weights are updated that is known as Selective Coefficient Update (SCU) [13], [14]. The structure adaptation algorithm can be applied on this SCU adaptive filters that leads to the closest possible performance to the full update algorithm. This is mentioned as Variable Structure Selective Coefficient Update (VS-SCU) algorithm in this paper. To evaluate the performance of the discussed adaptive algorithms under various noise environments and time varying acoustic channel the Signal to Noise Ratio (SNR) is fixed at various values during simulations. The main aim of this paper is to find an improved variable structure sub-filter based AEC performance in terms of echo removal, acoustic signal recovery, MSE improvement, faster convergence and reduced filter complexity.

\section{Problem Formulation}

A simplified model for monophonic AEC with adaptive time varying filters is shown in Fig. 1. The trauma of acoustic echo because of the coupling between the loud speaker and microphone is disturbing to users and causes a reduction in speech quality in due course of communication [15]. In this framework when the near-end generates speech, the signal travels to the far-end room over communication channel. The far end speaker if used in hands-free mode loudly relays the voice and microphone at that particular end catches various reflections along the direct path. The near-end subscriber collects several delayed versions of his own speech [1], [16]. If the system has remarkable delay the direct path from microphone to loudspeaker is observed as an echo to the near-end, though this path is not due to an acoustic reflection.

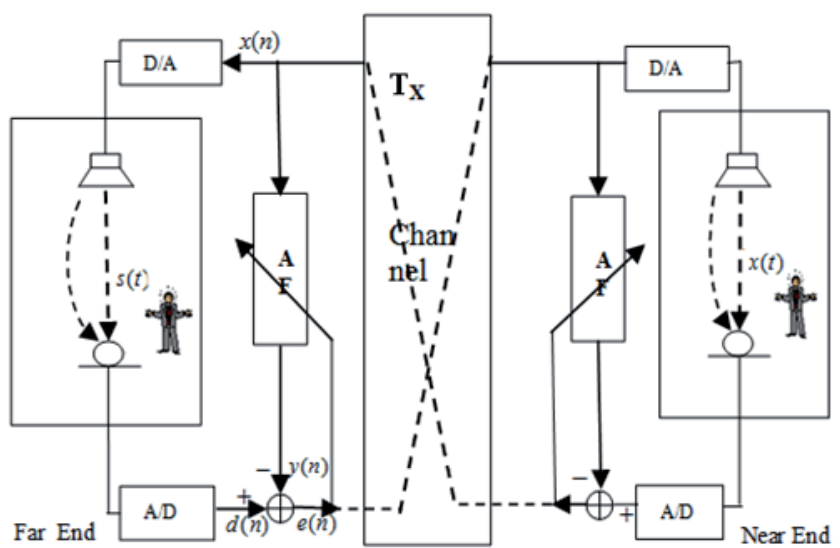

Fig. 1. AEC setup for both far and near end subscribers.
For error minimization, the filter impulse response must match acoustic response of the room environment. So the overall adaptive filter order has to dynamically vary and proceed towards a desired value in time varying environment to escalate the capability of echo control [9]. The tap-length or number of weights of the adaptive filter impulse response based AEC setup affects the overall performance of the echo cancellation. In many adaptive designs the tap-length is unfortunately set to a particular value creating the problem of unacceptable short and unwanted long filters. Less number of adaptive filter coefficients implicates shortage of taps for the system identification design which is termed as under-modeling. Whereas degraded convergence rate is the major drawback of large number of coefficients. So the length of adaptive filter should proceed towards the optimum value in dynamic situations to perfectly approximate the AEC accomplishment [10]. The requirement is to construct a suitable VSMSF based echo cancellation algorithm to reduce the acoustic echo as well as to minimize the structural and computational complexity of adaptive design in a dynamic room impulse response.

\section{Variable Structure MSF Based Acoustic Echo Canceller}

The LMS algorithm is basically used for adaptive weight update because of the intelligibility in execution and fewer calculations. However, when the input signal has large eigen value spread and the length of adaptive filter is large it converges slowly [14]. It is normally observed that LMS with fewer coefficients depicts improved convergence rate. Moreover, the time varying acoustic channel leads to further deterioration in performance. One way to minimize the slow convergence and calculative intensiveness of large adaptive filter issue in time domain is to use decomposition or MSF instead of using SLF [8-10]. The idea is based on partitioning the SLF into MSF. The decomposition technique allows efficient use of parallel processing which achieves increase in speed of the convergence. The logic of subdividing the input signal and weight vector into sub-vectors is demonstrated in [8], [9]. The concept is that signal realized by each branch of the multiple sub-filter cancels the signal in the corresponding echo path which results in fast convergence because the order of each sub-filter in MSF is much smaller as compared to order of SLF. Another way is to reduce the length of the fixed length adaptive filter to an optimum tap-length which reduces the structural complexity and due to reduced order increases the convergence [3].

In this paper work is done to use the variable taplength adaptive filtering and the MSF based partitioning at a time in an acoustic echo canceller to improve the convergence speed and to decrease the time as well as design complexity. The parallel MSF design dispenses the job of adjusting a long adaptive filter by one adaptive algorithm 
into lower length sub-filters updated independently [11]. Further different adaptive algorithms can be constructed depending upon characteristics of the generation of error signal. The error signal used for weight adjustment of filter can be found out at each stage of the sub-filter or it can be a common error obtained at the final stage of calculation named different error and common error respectively. The variable structure adaptive filter can be used in both these cases to find the optimum tap-length of each sub-filter. As each sub-filter collectively forms the parent filter, the dynamic tap-length adaptation algorithm finds a perfect structure which can be varied automatically as the echoed environment changes. The most effective current algorithm appoints both of this variable tap-length approach and the MSF parallel design to form an advanced echo canceller by suggesting VS-MSF-DEA and VS-MSF-CEA. The taplength optimization algorithm employed in most of these situations is the Fractional Tap-Length LMS (FT-LMS) or any of its modifications. The pseudo fractional tap-length that dynamically carries out the structure adaptation in an automatic time varying scenario was attained by the algorithm proposed in [3-5].

The well-known variable structure FT-LMS algorithm and its improved variants suffers from the flaw of random selection of important parameters like leakage factor, step size for weight adaptation, error width, length update stepsize that suppresses its applicability. The variable structure MSF based DEA and CEA as mentioned in existing research uses this length selection algorithm and depicts better performance compared to SLF [11]. Whereas both the DEA and CEA have their individual limitations of large steady state error and slower convergence respectively. In this manuscript research is carried out to design an improved variable structure algorithm and a new combined approach that holds the advantages of both VS-MSF-DEA and VS-MSF-CEA while eliminating the drawbacks. The SCU approach is also exercised on the proposed algorithm and it exactly approximates the results of a full update filter while diminishing the structural intricacy up to a notable extent.

\section{Proposed Variable Structure Adap- tive Acoustic Echo Cancellation Algorithms}

System identification is one of the most widely used applications of adaptive filters. In many identification frameworks the unknown plant impulse response varies dynamically according to the time varying environment. The old fashion of design suggests a fixed structure adaptive filter with predefined total number of weights to do the task of identification in these types of scenarios. The random tap-length initialization is made at a higher value to avoid the under-modeling issue where designers fall short of coefficients but on the other hand it increases the struc tural as well as computational complexity of the adaptive filter. Sometimes it generates adaptation noise due to mismatch of extra filter taps [4]. As the echoed enclosure can be varied for a person with a hands free mobile moving from a hall to inside the car, designing a fixed length filter for these types of issues cannot be considered as the best echo cancellation methodology. Hence, this motivates to design a variable structure adaptive filter where the taplength varies automatically and dynamically according to changing environment. In this paper an advanced algorithm is proposed which not only finds the dynamic structure more efficiently but also designs a better echo canceller with lower structural complexity, faster convergence and better tracing capabilities. The application of selective coefficient approach in context with the structure optimization algorithm results in the use of minimum filter coefficients and decreases the complexity further.

In the proposed variable structure algorithm the weight update of adaptive filter is not done with simple LMS or Normalized LMS (NLMS) algorithm. As the goal here is to search the optimized filter length hence, all the weight adaptation variables are made dependent on the instantaneous variable adaptive filter tap-length $P(n)$ obtained from the proposed fractional order estimation algorithm. In the existing algorithms the weight update is done with fixed parameters whereas on the other hand the filter length is made dynamic. It creates issues with stability of the overall system identification design and lowers the convergence. The modified NLMS based weight update algorithm mentioned in equation (1) provides inherent stability to the variable structure design [5], [12].

$W_{P(n)}(n+1)=W_{P(n)}(n)+\frac{\mu^{\prime}}{X_{p(n)}^{T}(n) X_{P(n)}(n)[2+P(n)]} X_{P(n)}(n) e_{P(n)}(n)$

where $W_{P(n)}, X_{P(n)}$ are the weight and input vectors pertaining to the tap-length $P(n) \mu^{\prime}$ is a step-size constant, $\sigma_{X}^{2}=X_{p(n)}^{T}(n) X_{P(n)}(n)$ is the variance of input signal. The desired signal $d(n)$ as shown in Fig. 1 for a simple AEC arrangement can be obtained as follows:

$$
d(n)=W_{P_{o p t}}^{T}(n) X_{P_{o p t}}(n)+t(n)
$$

where $W_{P_{o p t}}(n), X_{P_{o p t}}(n)$ are the weight and input vector pertaining to optimum tap-length $P_{\text {opt }}$ and $t(n)$ is the system noise. Similarly the error signal with respect to taplength $P(n)$ as shown in Fig. 1 is the difference between desired signal $d(n)$ and the adaptive filter output $y(n)$ which is equal to $W_{P(n)}^{T} X_{P(n)}(n)$,

$$
e_{P(n)}(n)=d(n)-y(n)
$$

Now the tap-length dependent time varying step-size $\mu_{P}(n)$ can be mentioned as follows: 


$$
\mu_{P}(n)=\frac{\mu^{\prime}}{\sigma_{X}^{2}[2+P(n)]} .
$$

Substituting step-size $\mu_{P}(n)$ in (1) the weight update equation is modified as follows:

$$
W_{P(n)}(n+1)=W_{P(n)}(n)+\mu_{P}(n) X_{P(n)}(n) e_{P(n)}(n) .
$$

This forms a tap-length dependent variable step NLMS algorithm where the step size depends on the instantaneous filter length. If the difference of the MSE output of any two consecutive coefficients of the adaptive filter falls below a very small positive value on further addition of weights to the total order, then it can be concluded that adding extra taps do not reduce the MSE.

Let's define $\Delta_{P}=J_{P-1}(\infty)-J_{P}(\infty)$ as the difference between the converged MSE when the tap-length is increased from $P-1$ to $P$. Now the optimum tap-length can be defined as $P_{\text {opt }}$ that satisfies

$$
\Delta_{P} \leq \delta \quad \text { for all } P \geq P_{o p t}
$$

where $\delta$ is a very small positive number that is fixed as per the system requirement [5]. The cost function for taplength selection can be defined as $\min \left\{P \mid J_{P-1}-J_{P} \leq \delta\right\}$. The issue of false optimum taplength sometimes creates confusion in the search of desired filter length. This pseudo-optimum tap-length can be one value or a set of values. Let there exist a positive integer $V$ that satisfies,

$$
V<P_{o p t} \text { and } \Delta_{V}<\delta
$$

where $V$ is called the pseudo-optimum tap-length. If the above condition is satisfied by a group of concatenated integer $V, V+1, \ldots, V-L+1$ then $L+1$ is called the width of the pseudo-optimum filter order. This tap satisfies the optimality condition but cannot be treated as the optimal filter length as it under-model the system. The issue of this pseudo-optimum tap-length can be removed by choosing a tap-length dependent variable error width $\Delta_{P}(n)$ which is discussed further in this section.

The steady state MSE is not available usually and can be found out by exponential averaging as follows:

$$
J_{P(n)}(n+1)=(1-H)\left(e_{P(n)}\right)^{2}(n+1)+H J_{P(n)}(n)
$$

where $H$ is the smoothing constant which controls the effective memory of the iterative process.

In this section an attempt is made to find a variable structure adaptive filter where the filter order or total number of adaptive weights $P(n)$ is dynamically decided according to the nature of the AEC framework. To find out the tap-length adaptation at first a smoothed estimated error can be obtained from [4], [6]

$$
\tilde{e}_{P(n)}(n)=(1-f) \sum_{i=0}^{n-1} f^{n-i} e_{P_{o p t}}(i)+t(i) f^{i}, 0<f<1
$$

where $n$ is the time index, $P_{\text {opt }}$ is the optimum tap-length which is initialized at zero at the beginning of tap-length adaptation, $e_{P_{o p t}}$ is the error signal at $P_{o p t}, t$ is the noise sample and $f$ is a forgetting smoothing constant. The performance of the squared smoothed estimated error can be simplified at the steady state as [6]

$$
E\left[\tilde{e}_{P(\infty)}(\infty)^{2}\right] \cong(1-f)(1-f)^{-1} \sigma_{t}^{2}
$$

where $P(\infty)$ is the steady state tap-length and it is assumed that the expectation of the square of smoothed estimated error signal approximates to the noise sample at the steady state. Now the algorithm for tap-length adaptation in a time varying environment is defined as [4]

$$
P_{n f}(n+1)=\left[P_{n f}(n)-K_{n}\right]+\left[\left(\tilde{e}_{P(n)}(n)\right)^{2}-\left(\tilde{e}_{P(n)-\Delta_{P}(n)}(n)\right)^{2}\right] \bar{K}_{n}
$$

where $P_{n f}(n)$ is the filter length that can take fractional values, $K_{n}$ is the leakage factor which prevents the taplength from increasing to an unexpectedly large value and factor $\bar{K}_{n}$ is the step size for tap-length adaptation. The variable error width parameter $\Delta_{P}(n)$ with respect to taplength $P(n)$ decides the bias between the unknown optimum tap-length $P_{\text {opt }}$ and the steady state tap-length $P(\infty)$ in a system identification framework. It removes the suboptimum values and finds the suitable best fit tap-length. A large value of $\Delta_{P}(n)$ produces large error width and brings heavy computational complexity whereas a small $\Delta_{P}(n)$ slows down the convergence and makes it difficult to overcome the suboptimum values. The steady state taplength is approximately equal to $P_{o p t}+\Delta_{P}(n)$ in order to maintain the trade-off between convergence and steady state error [4-6]. The variable error width $\Delta_{P}(n)$ depends on the smoothed estimated error variance $\tilde{e}_{P(n)}{ }^{2}(n)$ which can be expressed as [4-6]

$$
\begin{gathered}
\tilde{e}_{P(n)}{ }^{2}(n)=\rho \tilde{e}_{P(n)}{ }^{2}(n-1)+(1-\rho)^{-1} \tilde{e}_{P(n)}{ }^{2}(n-1), \\
\Delta_{P}(n)=\min \left(\Delta_{P, \max }, v \tilde{e}_{P(n)}{ }^{2}(n)\right)
\end{gathered}
$$

where $\rho$ is the smoothing parameter and $v$ is a constant set as per system requirements [4-6] and $\Delta_{P, \max }$ is the maximum permitted value of error spacing $\Delta_{P}(n)$. The steady state analysis of the proposed algorithm is given in this section. It also provides a general guideline for choice of error width parameter.

The steady-state Excess MSE (EMSE) of LMS can be defined as [5] 


$$
J_{e x}(\infty)=\frac{\mu(\infty) P(\infty) \sigma_{x}^{2} \sigma_{t}^{2}}{2-\mu(\infty) P(\infty) \sigma_{x}^{2}}
$$

where $\mu(\infty)$ is the steady state step-size at steady state tap-length $P(\infty), \sigma_{x}^{2}$ the variance of input signal and $\sigma_{t}^{2}$ is the noise variance which can be estimated in advance from noise sample $t(n)$. Again there is a need to search the steady state value of variable error width $\Delta_{p}(\infty)$ as follows:

$$
\Delta_{P}(\infty)=v\left(J_{e x}(\infty)+\sigma_{t}^{2}\right)=\frac{2 v \sigma_{t}^{2}}{2-\mu(\infty) P(\infty) \sigma_{x}^{2}} .
$$

Fixing the filter weights at the optimum value produces the minimum error output as follows:

$$
e_{\text {min }}(n)=t(n)+d(n)-W_{o p t}^{T} x(n) .
$$

Corresponding minimum output power is the minimum MSE (MMSE);

$$
J_{\text {min }} \cong E\left[e_{\text {min }}^{2}(n)\right]=\sigma_{t}^{2}+\varpi_{\text {min }}
$$

where parameter $\varpi_{\min }$ is obtained as,

$$
\varpi_{\text {min }} \cong \sigma_{d}^{2}-\mathrm{B}^{T} W_{\text {opt }} .
$$

Here $W_{\text {opt }}$ is the optimum set of filter weights, $\sigma_{d}^{2}=E\left[d^{2}(n)\right]$ is the variance of the desired signal, $B=E[X(n) d(n)]$ is the cross-correlation between input and desired signal. For arbitrary values of the weights, the total output error power consists of two components i.e. obtained as follows:

$$
\sigma_{e}^{2} \cong E\left[e^{2}(n)\right]=J_{\text {min }}+J_{e x}(n)
$$

where $J_{\min }$ is the MMSE and $J_{e x}(n)$ is the Excess MSE (EMSE). Applying (17) error variance $\sigma_{e}^{2}$ can be modified as follows,

$$
\sigma_{e}^{2} \cong \sigma_{t}^{2}+\varpi_{\min }+J_{e x}(n)
$$

Now

$$
\Delta_{P}(\infty)=\frac{2 v \sigma_{t}^{2}}{2-\frac{\mu^{\prime}}{[P(\infty)+2]} P(\infty) \sigma_{x}^{2}}=\frac{2 v \sigma_{t}^{2}}{2-\mu^{\prime}}
$$

Although $\Delta_{P}(n)$ is needed for different applications, whereas for a certain application it can be easily decided in advance according to the noise conditions. But the taplength which is assumed to be a fractional value in (11) should be rounded to nearest integer value. Hence, the optimized dynamic instantaneous filter length can be obtained as,

$$
\begin{gathered}
P(n+1)=\left\langle P_{n f}(n)\right\rangle \text { if }\left|P(n)-P_{n f}(n)\right|>\frac{K_{n}}{\bar{K}_{n}} \\
P(n) \quad \text { otherwise }
\end{gathered}
$$

where \langle\rangle operator signifies approximation of fractional order to the nearest integer value. In existing research works the value of leakage factor and adaptation step-size are based on setting a random leaky factor which limits its applicability to highly time varying environments. The parameters $\left(K_{n}, \bar{K}_{n}\right)$ should be set such that at the steady state it is close to zero to avoid under-modeling and large enough at the time of adaptation to avoid wandering issues. Thus there exists a need of variable leakage factor and adaptation step-size. In this work a unique method is implemented for setting these parameters.

$$
K_{n}=\min \left(K_{n, \max }, K_{n}(n+1)\right)
$$

where $K_{n, \max }$ is the maximum value of leakage factor. Again,

$$
\begin{gathered}
K_{n}(n+1)=\tilde{e}_{P}^{2}(n+1)\left(\tilde{e}_{P}^{2}(n+1)+\Delta_{p}(n)\right)^{-1} \\
\tilde{e}_{P(n)}(n+1)=f \tilde{e}_{P(n)}(n)+(1-f) e_{\min }(n+1)
\end{gathered}
$$

where $\tilde{e}_{P(n)}(n)$ is the smoothed estimated error with respect to $P(n)$. Similarly the adaptation step size depends on the bias between MSE values with a $\Delta_{p}(n)$ difference and $f$ is a forgetting smoothing constant. If the difference is more, then adaptation should be slow and vice versa. Again the adaptation step-size $\bar{K}_{n}$ can be obtained from its maximum limit with the help of a mathematical multiplicative smoothing parameter $\tau$ as follows [4], [5]

$$
\bar{K}_{n}=\min \left(\bar{K}_{n, \max }, \tau \bar{K}_{n, \max }\right)
$$

where

$$
\begin{aligned}
& \left.\tau=\left[\tilde{e}_{P(n)}(n)\right)^{2}-\left(\tilde{e}_{P(n)-\Delta_{P}(n)}(n)\right)^{2}\right] \\
& {\left[\left(\tilde{e}_{P_{o p t}(n)}(n)\right)^{2}+f\left[\tilde{e}_{P(n)}(n)\right)^{2}-\left(\tilde{e}_{P(n)-\Delta_{P}(n)}(n)\right)^{2}\right]^{-1}}
\end{aligned}
$$

\subsection{Variable Structure-MSF Based Different Error Algorithm}

Schematic for different error algorithm for AEC is given in Fig. 2. Each sub-filter shown in Fig. 2 has a dynamic structure with automatic tap-length varying ability instead of fixed structure. The sub-filters used in DEA, originate different error signals for adaptation which yields minimized coupling and enhances convergence speed [9]. Here, the error signals are independent on each other. In this case as the number of sub-filters increases there is a revamp in convergence but the steady state error behavior deviates from its ideal characteristics.

Let $X(n)=[x(n), x(n-1), \ldots \ldots \ldots \ldots \ldots .$.$] be the input$ vector. The $i^{\text {th }}$ dynamic sub-filter input vector can be represented as:

$$
X_{P(n), i}(n)=X[i P(n)+n] \forall i=0,1,2, \ldots, S-1
$$


where $S$ is the total number of sub-filters used in the design. The different error signals in case of VS-DEA for each sub-filter can be found out as follows:

$$
\begin{gathered}
e_{P(n), 0}(n)=d(n)-X_{P(n), 0}^{T}(n) W_{P(n), 0}(n), \\
e_{P(n), i}(n)=e_{P(n) i-1}(n)-X_{P(n), i}^{T}(n) W_{P(n), i}(n)
\end{gathered}
$$

where $P(n)$ is the dynamic tap-length of each sub-filter that is decided as per the variable structure algorithm, $d(n)$ is the channel output, $e_{P(n), i}(n), X_{P(n), i}(n)$ and $W_{P(n), i}(n)$ are the error, input and weight vectors respectively pertaining to variable tap-length $P(n)$ for the $i^{\text {th }}$ dynamic sub-filter. The weight adaptation algorithm for VS-MSF-DEA can be shown as:

$$
\begin{aligned}
& W_{P(n), i}(n+1)=W_{P(n), i}(n)+\mu_{P(n), i} e_{P(n), i}(n) X_{P(n), i}(n) \\
& \forall i=0,1,2, \ldots, S-1
\end{aligned}
$$

where $\mu_{P(n), i}$ is the $i^{\text {th }}$ dynamic sub-filter step-size pertaining to variable tap-length $P(n)$.

\subsection{Variable Structure-MSF Based Common Error Algorithm}

The VS-MSF-CEA does exactly the reverse operation of different error variant discussed earlier. It uses only one common error for weight update of each sub-filter used in MSF division. It achieves better MSE compared to DEA but convergence slows down because of the coupling of weight update equations. The error signal in this case is dependent on all other errors. The common error signal updates each sub-filter individually by an adaptive algorithm [9-11]. The steady state error is less here and the sub-filters used in the structure find their optimum taplength employing the dynamic tap-length selection algorithm. The dynamic structure of common error schematic is shown in Fig. 3. The adaptation algorithm for VS-MSFCEA can be obtained as mentioned below:

For each instant of time $n=0,1,2, \ldots$ and desired signal $d(n)$, the common error signal $e(n)$ is obtained as

$$
\begin{gathered}
e(n)=d(n)-\sum_{i=0}^{S-1} X_{P(n), i}^{T}(n) W_{P(n), i}(n), \\
W_{P(n), i}(n+1)=W_{P(n), i}(n)+\mu X_{P(n), i}(n) e(n) \\
\forall i=0,1,2, \ldots, S-1
\end{gathered}
$$

\subsection{Variable Structure-MSF Based Combined Error Algorithm}

The aim of a researcher is to invariably find an efficient echo canceller that results in better convergence and MSE outcomes under various noise conditions. But practically it is not possible to frame such MSF design that is completely different form VS-MSF-DEA and VS-MSF-

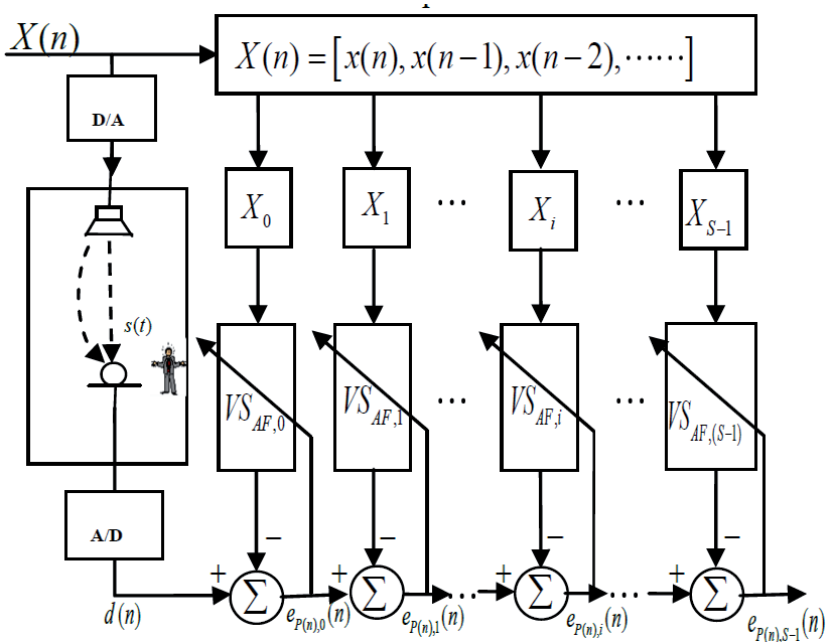

Fig. 2. Variable structure MSF based DEA design for AEC.

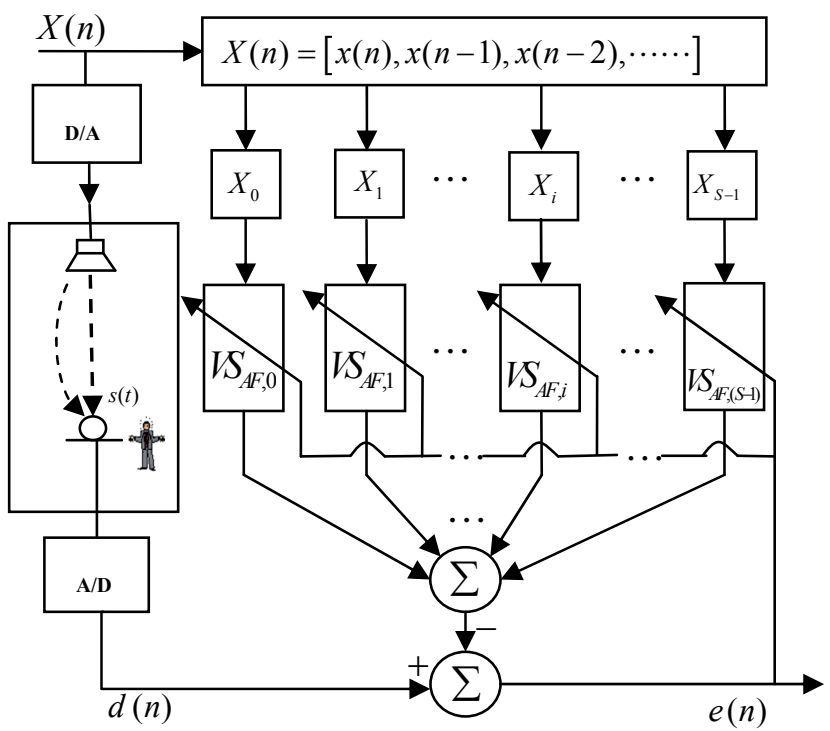

Fig. 3. Variable structure MSF based CEA design for AEC.

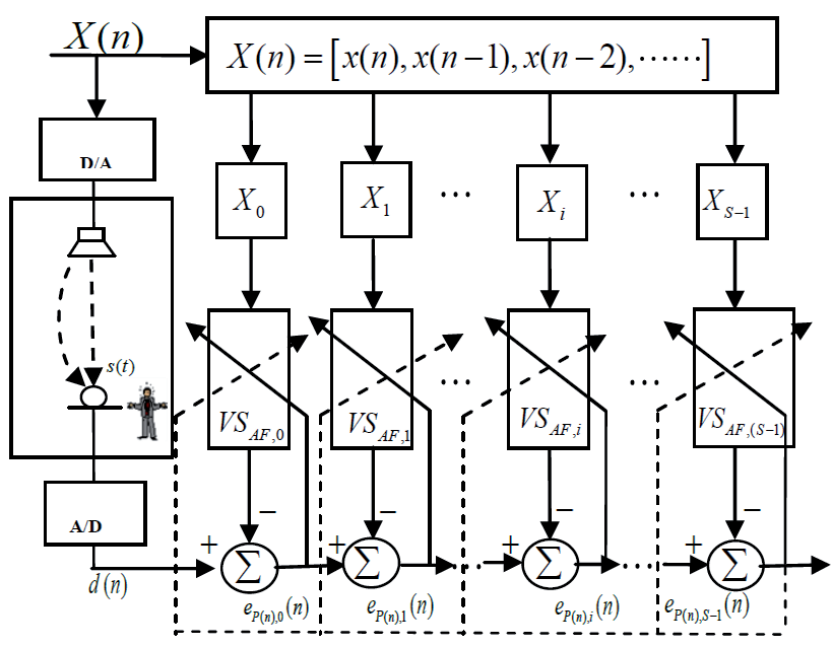

Fig. 4. Proposed variable structure MSF based COEA design for AEC.

CEA. A combination of both of these algorithms can be obtained which provides a trade-off between the conver- 
gence rate and the steady state error according to requirement. The combined error MSF based structure is shown in Fig. 4. A trade-off parameter alpha $\alpha$ is set appropriately to achieve nearly optimized results. To attain appropriate performance parameter selection plays an important role. This decides the contribution of each algorithm i.e. VSMSF-DEA and VS-MSF-CEA in overall performance of the echo canceller. The trade-off can adjust both convergence and steady-state error but it is application specific [9], [11]. The value of $\alpha$ lies between 0 and 1. The tradeoff $\alpha$ contributes to the adaptation of VS-MSF-DEA and $1-\alpha$ to the adaptation of VS-MSF-CEA in the combined algorithm. Hence, in those applications where convergence is the requirement over steady state error $\alpha$ selection goes close to 1 and for better steady state error requirement $\alpha$ selection approximates to 0 or $<<1$. In this paper it is fixed at 0.5 as per the simulation framework to get a perfect adjustment between MSE and convergence rate. Hence, a user by following the proposed structure can fulfill the needs of echo cancellation application by varying the trade-off as per the required specification. The adaptation algorithm for Combined Error AEC is obtained as follows:

$W_{P(n), i}(n+1)=W_{P(n), i}(n)+X_{P(n), i}(n)\left[\alpha \mu_{P(n), i} e_{P(n), i}(n)+(1-\alpha) \mu e(n)\right]$ $0 \leq \alpha \leq 1, \forall i=0,1,2, \ldots, S-1$

where $e_{P(n), i}(n)$ is the different error signal for the $i^{\text {th }}$ dynamic sub-filter pertaining to its optimum tap-length $P(n)$ and $e(n)$ is the common error signal.

\subsection{Variable Structure-MSF Based Selective Coefficient Update}

In AEC applications the computational complexity of adaptive algorithm and structural complexity of overall filter design increases. This happens as a result of high requirement of filter coefficients. The total number of adaptive weights contributes to the computational intricacy of adaptive algorithms. They are found to be directly proposed in AEC applications. This motivates research on the selective coefficient adaptive algorithm which decreases the weight requirement up to a great extent by employing a selection matrix [13].

Updating the entire set of weights in an adaptive filter is exorbitant in terms of storage and calculative requirements hence, the computational complexity needs to be reduced. The work of a researcher in this case is to lessen the entanglement in filter design. There are basically two strategies to optimize the set of filter coefficients. One is variable structure algorithm which has been already discussed in the previous section and another strategy is to adapt selected set of filter weights rather than all at a time. In this manuscript both the strategies are used together to lessen the weight requirement to the maximum extent without substantial reduction in MSE performance. The adaptive filter can find a better convergence and taper computational cost if the total number of weights is por- tioned into smaller selected blocks of coefficients each updated by applying the variable tap-length dynamic algorithm which is referred as VS-SCU in this paper.

It is perceived that the number of weights adapted in each and every iteration is amortized in SCU approach but it comes at the cost of performance mortification. Hence, the VS-SCU approach has to find a method in which it can reduce total number of weights updated per iteration with performance deterioration as small as possible. VS-SCU enables the computational cost for modifying the weights of an adaptive filter to be decreased without necessarily lowering the filter length. In VS-SCU, let $Q$ weights out of $P(n)$ optimum weights are updated. From a set of $P(n)-b y-P(n)$ coefficients selection matrix $M$ is chosen such that only $Q$ values will have 1's as diagonal element where weights are needed to be updated. The weight update equation for VS-SCU is obtained as follows:

$W_{P(n)}(n+1)=W_{P(n)}(n)+M \mu_{P(n)} X_{P(n)}(n) e_{P(n)}(n) ; \quad n=0,1,2 \ldots .$.

The criterion for finding the selection matrix $M$ is based on [13], [14]. The above equation shows SCU for variable structure single long filters. The same can be done for each sub-filter in MSF based designs. The main motive of this selective coefficient approach is to identify and update with those selected coefficients which result in nearly same accomplishment as that of full update. But this should occur with minimized performance loss in terms of MSE and echo cancellation.

\section{Simulation and Results}

The Matrix Laboratory (MATLAB) platform has been chosen for simulation purpose. For better comparison with the existing research work the simulation of the room impulse response is done nearly same as that of [3-5], [9], [11]. The room impulse response is measured for an enclosure of dimension $12 \times 12 \times 8 \mathrm{ft}^{3}$ using an ordinary loudspeaker and an omnidirectional microphone kept at a distance of 1 feet. It has been sampled at $8 \mathrm{kHZ}$. The channel response is obtained by applying a stationary Gaussian stochastic signal with zero mean and unit variance as input on the measured impulse response. The tap-length of SLF is fixed at 2000 and the length of each sub-filter for $S=5$ is 400. The adaptation step size is chosen to be 0.0004 for SLF and 0.00055 for each sub-filter. The value of trade-off parameter $\alpha$ is fixed at 0.5 .

The FT-LMS algorithm with fixed error width achieves the optimum tap-length for each sub-filter as shown in Fig. 5 over 200 Monte-Carlo runs and 10,000 iterations but results in under-modeling the system. On the other hand the proposed variable structure algorithm best adjusts the system performance in comparison to the VTVSLMS [4] and FT-LMS algorithm [3]. It reduces the overall weight requirement to nearly 1500 instead of a fixed selection of 2000 as each sub-filter carries optimum 


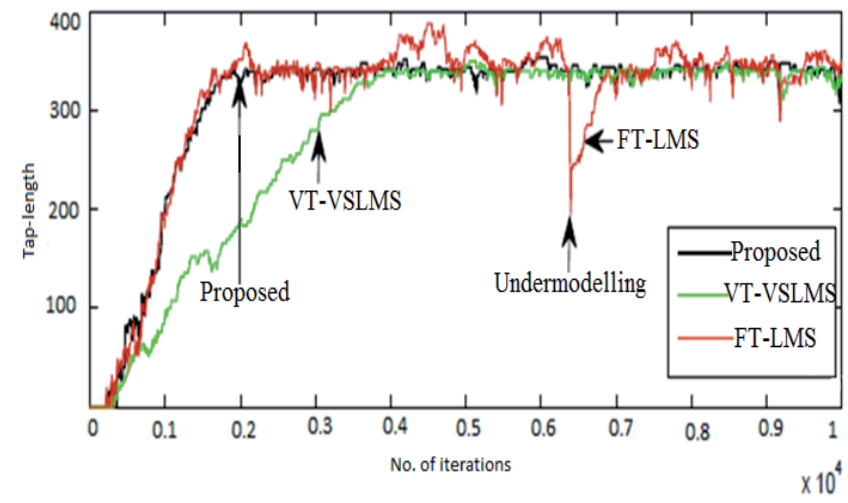

Fig. 5. Comparison of proposed VS tap-length selection algorithm with FT-LMS and VT-VSLMS.

tap-length of 300 with the application of proposed structure adaptation algorithm. The simulation is carried out for both low and high noise environments to judge the dynamic capability of the algorithm.

In Fig. 6 for low noise environment SNR is kept at $30 \mathrm{~dB}$. It can be observed that the VS-MSF-DEA based AEC shows better convergence speed over all the other variable structure AEC algorithms but it lacks in MSE performance. Similarly the VS-MSF-CEA improves the

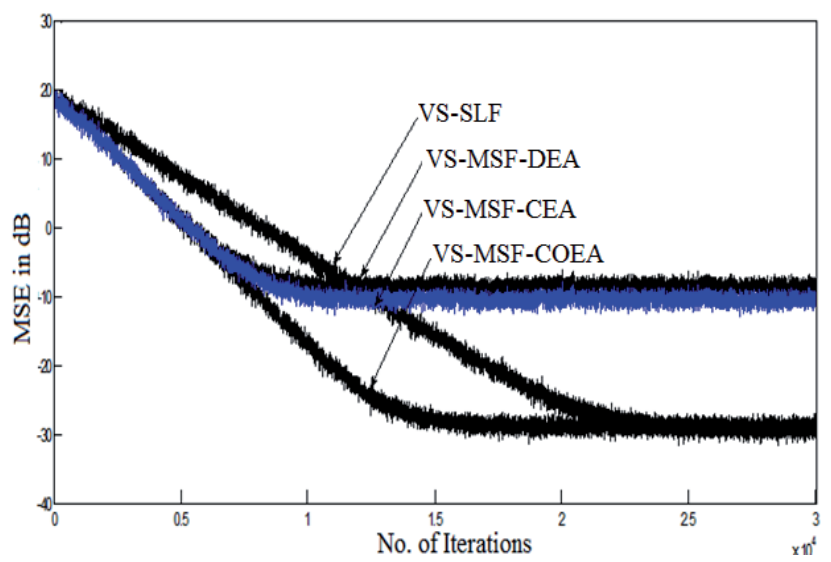

Fig. 6. Comparison of MSE between proposed VS echo cancellation algorithms at $\mathrm{SNR}=30 \mathrm{~dB}$ (low noise).

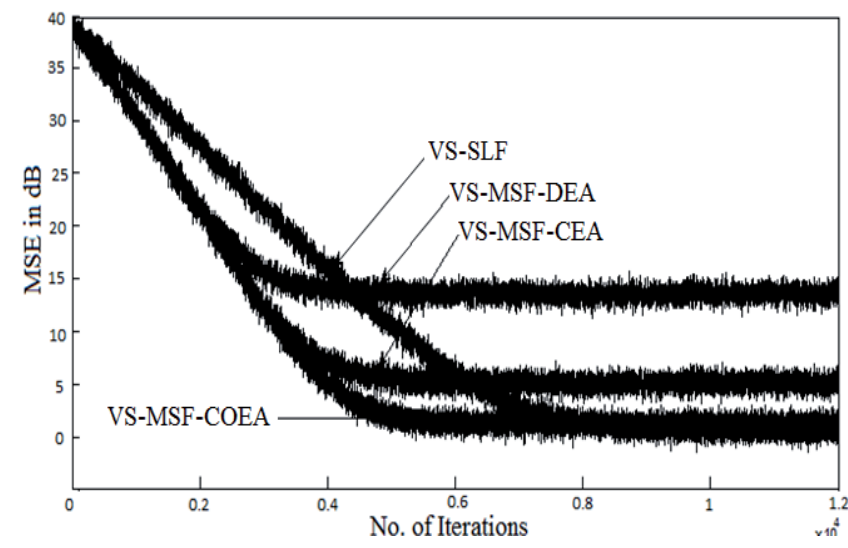

Fig. 7. Comparison of MSE between proposed VS echo cancellation algorithms at $\mathrm{SNR}=0 \mathrm{~dB}$ (high noise).
MSE performance but has a slow convergence. Hence, the proposed VS-MSF-COEA maintains perfect trade-off between the two algorithms proposed in [9], [11] and outperforms the VS-SLF also. The same result is obtained as shown in Fig. 7 when SNR is kept at $0 \mathrm{~dB}$ for high noise case over 30000 iterations and 200 independent runs. The SCU algorithm is applied to SLF and VS-MSF-COEA with a $30 \%$ of the full update coefficients and the results are shown in Fig. 8. It exactly replicates the full-update filter performance while reducing the structural complexity up to a great extent.

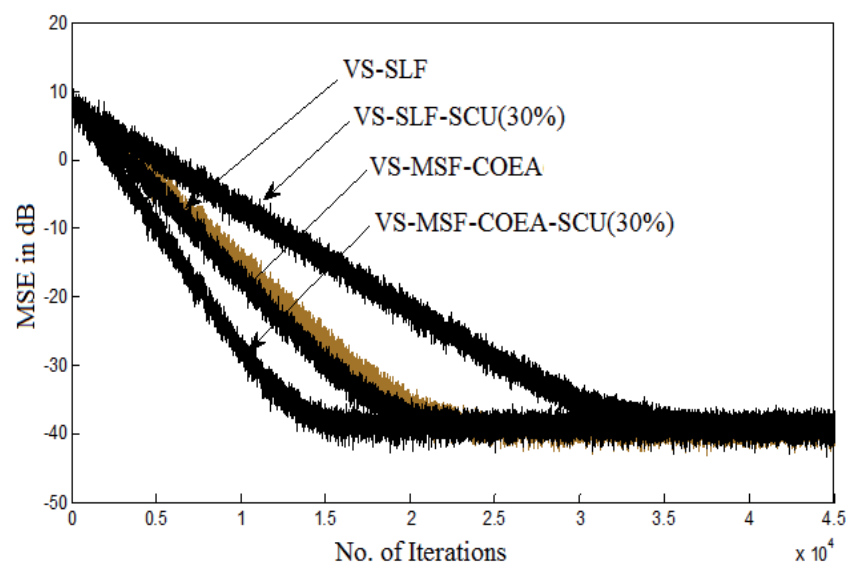

Fig. 8. Comparison of MSE between SCU based VS-SLF and proposed algorithm at $\mathrm{SNR}=40 \mathrm{~dB}$.

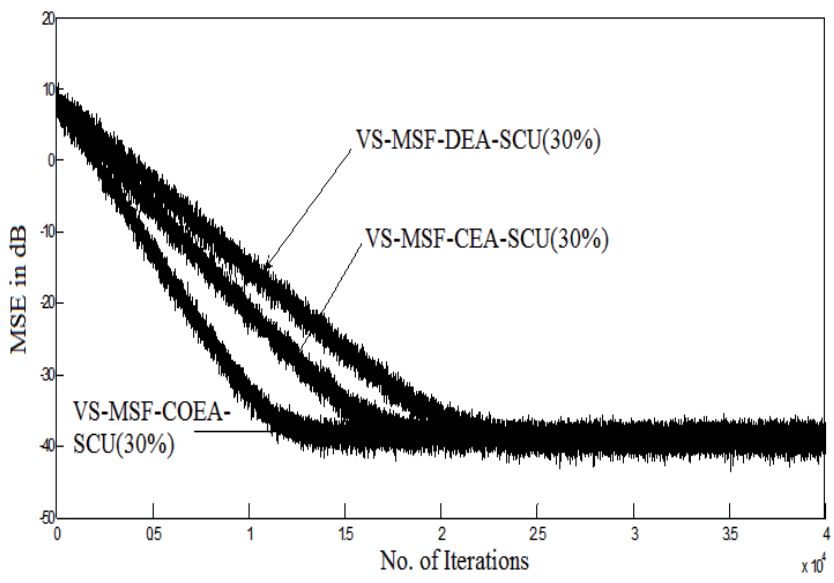

Fig. 9. MSE performance comparison of all SCU (30\%) based VS echo cancellation algorithms at $\mathrm{SNR}=40 \mathrm{~dB}$

In Fig. 9 the plot shows the SCU with 30\% coefficient update that is applied to all variants of VS-MSF algorithms for echo cancellation. In this case the full-update tap-length can be considered as 1500 instead of 2000 as the structure adaptation algorithm has already been applied before the application of SCU method. It can be noticed that the SCU variant of the proposed VS-MSF-COEA depicts improved performance than its counterparts. The filter design is done with a mere 500 coefficients rather than 2000 filter weights. This claims that the proposed algorithm reduces the structural complexity up to a great extent and overcomes the drawback of VS-SLF, VS-MSFDEA and VS-MSF-CEA. 
Tracking is a steady state phenomenon which means the ability to follow statistical variations in a non-stationary environment. The tracking behavior indicates how efficiently the adaptive filter can follow the changes in the room impulse response. In case of AEC, the echo canceller scrutinizes the changing impulse response of the echo path. The movements of the local speaker lead to large changes of the room impulse response.

The acoustic echo canceller has to adapt the changing channel condition. The tracking effect is examined by switching from one room impulse response to other. If the length of the filter is small it is able to change the dislocation but if the filter length is larger it takes more time to track. In this paper the proposed dynamic length adaptation adaptive algorithms offers improved tracking capability in a non-stationary environment.

The tracking effect can be judged by enclosure dislocation after starting the MSE adaptation for some iterations. The result shown in Fig. 10 depicts the system model of tracking for all the discussed echo cancellation algorithms. It can be observed that the tracking capability of DEA and CEA is better in comparison to SLF while analyzing with a variable structure but the convergence performance of proposed algorithm is better than all its predecessors. The tracking performance of the VS-MSF-COEA and VS-MSF-COEA-SCU (40\%) are compared as shown



Fig. 10. Tracking performance comparison of VS echo cancellation algorithms at $\mathrm{SNR}=40 \mathrm{~dB}$.

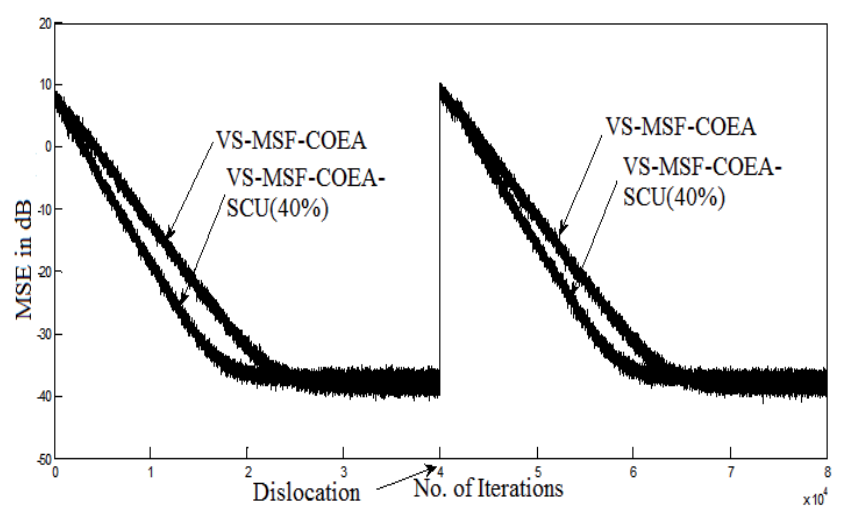

Fig. 11. Tracking performance comparison of proposed algorithm with its SCU (40\%) based counterpart at $\mathrm{SNR}=40 \mathrm{~dB}$.

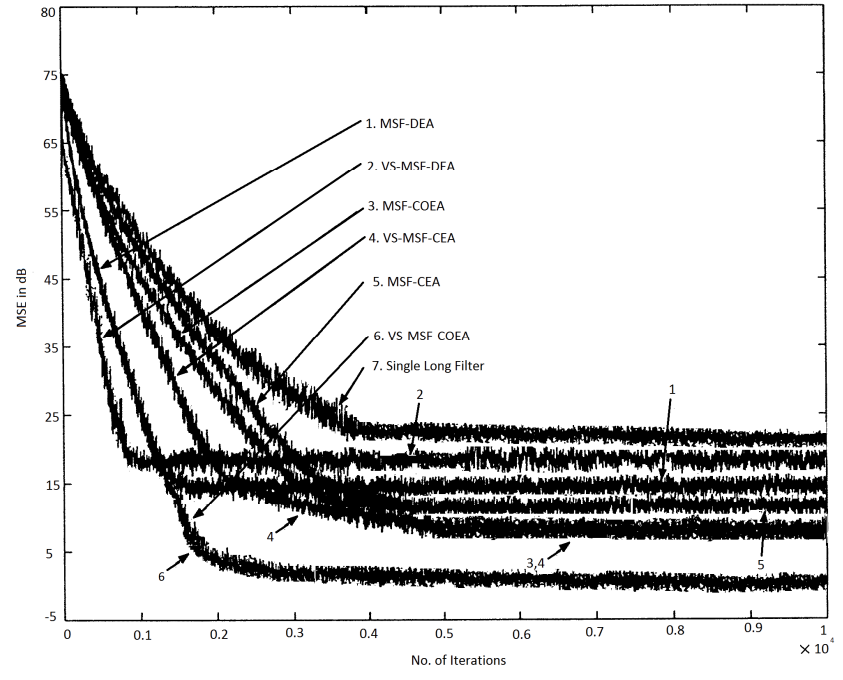

Fig. 12. Performance comparison of fixed tap-length MSF and VS-MSF acoustic echo cancellation algorithms at $\mathrm{SNR}=0 \mathrm{~dB}$.

in Fig. 11. The results are obtained by averaging over 100 independent runs. The SCU is used for adaptive filter where only 800 weights of adaptive filter is being updated in each iteration which corresponds to $40 \%$ of the long filter having 2000 coefficients. It nearly replicates the full update performance with 1200 fewer filter weights.

In Fig. 12 the performances of all available MSF based algorithms along with their proposed variable structure variants are studied at a high noise environment where SNR is fixed at $0 \mathrm{~dB}$. The variable structure approach improves the convergence speed for MSF-DEA, MSFCEA and MSF-COEA but depicts little compromise in MSE performance in different and common error designs. This occurs due to a reduction in total number of weights for the overall design and unavailability of trade-off parameter to monitor the performance. On the other hand the proposed VS-MSF-COEA design best adjusts the system execution compared to all its counterparts.

The VS-MSF-COEA comes out as the most efficient echo cancellation algorithm because it achieves optimum performance with a less cost of construction due to minimum requirement of optimum filter weights as decided by the variable structure algorithm. The SCU approach can be applied on this design to reduce the complexity further. A proper selection of percentage of coefficients in VSMSF-COEA-SCU can make it a better choice.

\section{Conclusion}

The VS-MSF-COEA algorithm introduced in this paper and its SCU variant has lower complexity, faster convergence rate and good tracking capabilities. The proposed VS-MSF-COEA algorithm is analyzed to find the steady state performance and to set variable parameters for structure adaptation. The proposed algorithm has shown consistently superior performance over the existing VS-MSFDEA, VS-MSF-CEA algorithms in terms of convergence 
speed, steady state error, tracking and achieves all of this with a minimized structural complexity with less number of optimized selected coefficients. The algorithm may further be analyzed for applications like channel equalization, noise reduction etc. A different analysis with notable performance escalation can also be carried for applications severely affected by high noise elements. Further research can be carried out on the selective update of filter weights based VS-MSF-COEA-SCU algorithm for AEC as there is an issue about the percentage of coefficient selection for time varying applications.

\section{References}

[1] SCHÜldT, C., LINDSTROMB, F., LI, H., CLAESSON, I. Adaptive filter length selection for acoustic echo cancellation. Signal Processing, 2009, vol. 89, no. 6, p.1185-1194. DOI: 10.1016/j.sigpro.2008.12.023

[2] GUPTA, V. K., CHANDRA, M., SARAN, S. N. Acoustic echo and noise cancellation system for hand-free telecommunication using variable step size algorithms. Radioengineering, 2013, vol. 22 , no. 1 , p. $200-207$.

[3] GONG, Y., COWAN, C. F. N. An LMS style variable tap-length algorithm for structure adaptation. IEEE Transactions on Signal Processing, 2005, vol. 53, no. 7, p. 2400-2407. DOI: 10.1109/TSP.2005.849170

[4] KAR, A., CHANDRA, M. Pseudo-fractional tap-length learning based applied soft computing for structure adaptation of LMS in high noise environment. In Soft Computing Techniques in Engineering Applications, p. 115-129, Springer-Verlag, Germany, 2014. DOI: $10.1007 / 978-3-319-04693-8 \_8$

[5] KAR, A., CHANDRA, M. A minimized complexity dynamic structure adaptive filter design for improved steady state performance analysis. International Journal of Computational Vision and Robotics, 2014, vol. 3, no. 4, p. 326-340. DOI: 10.1504/IJCVR.2013.059100

[6] LI, L. Adaptive algorithms and variable structures for distributed estimation. Ph.D. Thesis, Advanced Signal Processing Group, Loughborough University, 2009.

[7] WANG, X., SHEN, T., WANG, W. An approach for echo cancellation system based on improved NLMS algorithm. In Proceedings of IEEE Wireless Communications, Networking and Mobile Computing Conference. Shanghai (China), 2007, p. 2853 to 2856. DOI: 10.1109/WICOM.2007.708

[8] SHARMA, R. N., CHATURVEDI, A. K., SHARMA, G. Acoustic echo cancellation using multiple sub-filters. In Proc. of IEEE Conf. on Convergent Technologies for the Asia-Pacific Region TENCON 2003. Bangalore (India), 2003, p. 393-396. DOI: 10.1109/TENCON.2003.1273352

[9] BARIK, A., MURMU, G., BHARDWAJ, T. P., NATH, R. LMS adaptive multiple sub-filters based acoustic echo cancellation. In Proceedings of the IEEE International Conference on Computer and Communication Technology. Allahabad (India), 2010, p. 824 to 827 . DOI: $10.1109 /$ ICCCT.2010.5640392

[10] NATH, R. Adaptive echo cancellation based on a multipath model of acoustic channel. Ph.D. Thesis. Dept. of Electrical Engineering Indian Institute of Technology Kanpur, UP, India, 2005.

[11] KAR, A., CHANDRA, M. Dynamic tap-length estimation based low complexity acoustic echo canceller. In Proceedings of the IEEE International Conference on Emerging Trends in Science, Engineering and Technology. Trichy (India), 2012, p. 339-343. DOI: $10.1109 /$ INCOSET.2012.6513929
[12] WIDROW B., STERNS, S. D. Adaptive Signal Processing. Prentice Hall, Englewood Cliffs, NJ,1985.

[13] MAYYAS, K., ABOUNASR, T. Reduced-complexity transformdomain adaptive algorithm with selective coefficient update. IEEE Transactions on Circuits and Systems-II: Express Briefs, 2004, vol. 51, no. 3, p. 136-142. DOI: 10.1109/TCSII.2003.822437

[14] MAYYAS, K. New transform-domain adaptive algorithms for acoustic echo cancellation. Digital Signal Processing, 2003, vol. 13, no. 3, p. 415-432. DOI: 10.1016/S1051-2004(03)00004-6

[15] KELlERMANN, W. Echo Cancellation. Handbook of Signal Processing in Acoustics. Springer-Verlag, Germany, vol. 1, p. 883-895, 2009.

[16] YU, H., LIU, Z., LI, G. A VSLMS style tap-length learning algorithm for structure adaptation. In Proceedings of IEEE International Conference on Communication Systems. Guangzhou (China), 2008, p. 503-508. DOI: 10.1109/ICCS.2008.4737235

\section{About the Authors ...}

Asutosh KAR is working in the Dept. of Electronics \& Telecommunication Engineering at IIIT Bhubaneswar, India. Dr. Kar has gained teaching and research experience in various areas of signal, image and speech processing. He received his Bachelors in Technology in Electronics and Communication Engineering from B.P.U.T, India and Masters in Signal Processing and Control from NIT Hamirpur, India holding the first position in his branch. His Ph.D is from BIT Mesra, India and his research area includes digital signal processing, advanced signal processing, filter theory and image processing. He has published more than 40 research papers in the area of communication, signal and image processing at international levels. He is a guest editor of IJSISE, Interscience and review, editorial board member of IJIPVS, IJESS, Interscience. He has organized special sessions in IEEE SPIN-14, Springer ICACNI-14, IEEE ICACCI-14, IEEE MEDCOM-14 and Springer FICTA-14. He has also worked as a track chair in ETES14 and TPC member in IEEE SSCI-14, USA. He is in the review committee of many international and national conferences. He has received honor on behalf of President of India thrice from All India Radio for high quality technical presentation. He has guided P.G and U.G students in their project work. He is a member of IEEE USA, IACSIT, IRNet, IIRJC.

Mahesh CHANDRA received B.Sc. from Agra University, Agra, India in 1990 and A.M.I.E. from I.E.I., Kolkata, India in winter 1994. He received M.Tech. from J.N.T.U., Hyderabad in 2000 and Ph.D. from AMU, Aligarh in 2008. Presently, he is working as an Associate Professor in the Dept. of Electronics \& Communication Engineering at Birla Institute of Technology Mesra, Ranchi, India. He has published more than 110 research papers in the area of speech, signal and image processing at national/ international levels. He has guided $07 \mathrm{Ph} . \mathrm{D}$. students and 20 M.Tech students for their research work. He is a member of IEEE USA, ISTE India and Fellow of IETE, India. He has delivered a number of keynote talks and chaired many sessions at national/ international levels. 